\title{
Ergonomía Ambiental e lluminación Integrativa. Desarrollo de créditos para un Sistema de Etiquetado Energético-Ambiental de Edificios No Residenciales.
}

\section{Environmental Ergonomics and Integrative Lighting. Development of credits for an Energy-Environmental Labeling System for Non- Residential Buildings.}

Presentación: 06/10/2020

\author{
Doctorando: \\ Verónica J. Ruiz \\ Instituto Ambiente, Hábitat y Energía (INAHE) - Argentina \\ vruiz@mendoza-conicet.gob.ar
}

Director/a:

Roberto G. Rodriguez

Co-director/a:

Andrea E. Pattini

\section{Resumen}

A partir del análisis de distintos sistemas de Etiquetado-Energético Ambiental de Edificios (EEAE), en particular sus créditos específicos de Iluminación, el presente plan de trabajo doctoral busca proponer y validar un método de acreditación de iluminación integrativa, desde la perspectiva antropocéntrica propia de la ergonomía ambiental. Los resultados iniciales de este análisis se permiten delinear una taxonomía del andamiaje metodológico, organizacional y regulatorio asociado al EEAE

Palabras clave: Ergonomía Ambiental - Indicadores - Certificación Energética- lluminación Integrativa

\begin{abstract}
Based on the analysis of different Building Environmental Energy-Labeling (EEAE) systems, in particular their specific lighting credits, this doctoral work plan seeks to propose and validate an integrative lighting accreditation method, from the anthropocentric perspective typical of environmental ergonomics. The initial results of this analysis allow the delineation of a taxonomy of the methodological, organizational and regulatory scaffolding associated with the EEAE.
\end{abstract}

Keywords: Environmental ergonomics - Indicators - Energetic Certification - Integrative Lighting 


\section{Introducción}

La propuesta del presente plan de trabajo se origina en el trabajo final de Especialización en Desarrollo Sustentable del Hábitat Humano (UTN FRM), titulado "Iluminación sustentable. Diagnóstico y propuesta de intervención. El caso de una biblioteca pública.", con la tutoría del director de esta tesis. De aquí surge la necesidad de avanzar en el conocimiento de los efectos de la iluminación en la salud de los ocupantes de espacios interiores laborales, y de contar con indicadores y métodos de caracterización, evaluación, certificación y/o acreditación de estos efectos.

Uno de los mayores desafíos que actualmente enfrenta la humanidad se relaciona con la degradación ambiental, el sobreuso de recursos limitados y el cambio climático (Stern, 2006). Las potenciales consecuencias para la vida en nuestro planeta están impulsando un cambio de paradigma en cuanto a cómo vivimos, trabajamos y consumimos y exigen acciones para prevenir y mitigar el daño causado, propiciando un desarrollo sustentable. La respuesta a este desafío desde la perspectiva del entorno construido fue el diseño de edificios verdes (del inglés Green Buildings). La aparición en Inglaterra del Building Research Establishment Environmental Assessment Method (BREEAM) durante la década de 1990 fue la primera iniciativa para establecer un método objetivo y abarcador para evaluar en simultaneo un amplio rango de consideraciones ambientales en relación a criterios explícitos y resumirlos en una medida global de desempeño ambiental, y que dicho valor obtenido constituya una etiqueta de desempeño ambiental. El objetivo del etiquetado ambiental de edificios (EEAE) fue alentar un cambio en la industria de la construcción y transformar el mercado, que vería un incremento del valor real de edificios con mejores atributos ambientales certificados por dicha etiqueta (Cole, Howard, Ikaga \& Nibel, 2005). El campo del EEAE ha madurado desde entonces, y las últimas dos décadas han visto el rápido incremento de métodos disponibles en todo en el mundo (e.g. LEED Estados Unidos, TGBRS - India; CASBEE - Japón; NABERS, Australia, etc.). Actualmente existe un amplio abanico de métodos de EEAE que abordan diferentes aspectos del desarrollo sostenible, diseñados para diferentes tipos de proyectos. A marzo de 2010, Nguyen \& Altan (2011) contabilizaron 382 herramientas de software registradas para evaluar eficiencia energética, energías renovables y sustentabilidad edilicia. En relación con la Argentina, aún no hay una apropiación significativa de sistemas de EEAE.

Inicialmente el desarrollo de métodos de EEAE fue esencialmente un ejercicio de estructuración de consideraciones y conocimiento existente, considerando la practicidad de su aplicación. Estos métodos fueron concebidos como voluntarios y motivacionales en su aplicación. Por otro lado, la autoridad gradualmente comienza a consultar estas herramientas para establecer requerimientos legales mínimos de desempeño ambiental para nuevos proyectos. Con varios países seleccionando o desarrollando sus propios métodos de EAE, es evidente el proceso de internacionalización del EAE. En 1997, el comité técnico 59 (Building Construction) de la ISO estableció un grupo ad hoc, formalizado como el Sub Comité ISO TC59/SC17 Sustentabilidad en la Construcción de Edificios, cuyo alcance incluye a los métodos de EAE. Este subcomité ha sido responsable de la publicación de nueve normas ISO y cuenta con seis más en desarrollo. Por su parte, en la Argentina se encuentra en fase de pruebas piloto la Norma IRAM 11900 de etiquetado ambiental de viviendas (Vagge \& Czajwoski, 2012).

En 2007, la población mundial pasó a ser más urbana que rural, y se estima que esta proporción seguirá creciendo (SaraviaPinilla, Daza-Beltrán \& García-Acosta, 2016). En promedio, tanto para países industrializados como en desarrollo, las personas pasan entre el 80-90\% del tiempo dentro de espacios construidos (Hanlon, 2007; Klepeis et al, 2001) que necesitan ser calefaccionados, refrigerados, ventilados e iluminados, lo cual requiere del uso de energía. En particular los edificios del sector servicios requieren proporcionalmente más energía que en acondicionamiento ambiental que aquellos del sector residencial. En ese sentido, el Panel Intergubernamental de Cambio Climático (Schweizer, 2007) destacó que una significativa mitigación en el consumo energético puede lograrse con mejores diseños edilicios, estando el mayor potencial de ganancia en los países en desarrollo (Gibberd, 2005). Esto indica la pertinencia del presente plan de trabajo en el actual contexto energético de nuestro país.

Sin embargo, requerimientos de sustentabilidad pueden entrar en conflicto con aspectos ergonómicos de calidad ambiental interior (Thatcher \& Milner, 2014; Hirning, Isoardi \& Cowling, 2014; Khoshbakht, Gou, Lu, Xie \& Zhang, 2018). Y es allí donde la ergonomía está en posición de jugar un rol clave en el diseño sustentable de ambientes interiores (Hedge \& Dorsey, 2013; Rodriguez, Lasagno, Monteoliva, Yamín \& Pattini, 2017), coadyuvando al desarrollo de la actividad laboral en forma eficaz, eficiente y con confort. A partir del conocimiento de las capacidades y limitaciones humanas, y de las demandas impuestas por el entorno y la actividad, la ergonomía busca alcanzar un ambiente construido que impacte positivamente en el consumo energético, el desempeño laboral y la salud humana. La relación entre sustentabilidad y ergonomía ha sido postulada desde la última década del siglo XX (Moray, 1995), suscitando mayor interés desde la primera década de este siglo, a partir de los desarrollos de la Eco- Ergonomía (Brown, 2007), Ergo-ecología (2012), Green Ergonomics (Tatcher, 2013) y Factores Humanos y Sustentabilidad (Zink \& Fischer, 2013). 
Cedeño-Laurent y colaboradores (2018) afirman que más allá de una reducción del uso de energía y un mayor valor de mercado, la principal fortaleza del EEAE puede ser la consecución de entornos construidos interiores más saludables, permitiendo a la sustentabilidad edilicia alcanzar su potencial completo como una herramienta de transformación de salud pública. Más aun, estos autores postulan que el EEAE es la base crítica de investigación dentro de un nuevo marco de la arquitectura sustentable, basada en el capital humano. Del amplio abanico de factores que aborda el EEAE, esta investigación se acotará solamente a los aspectos lumínicos no visuales. Los objetivos tradicionales de la iluminación arquitectónica incluyen la provisión de luz que: i) sea óptima para el rendimiento visual; ii) sea visualmente confortable; iii) permita la apreciación estética del espacio; y iv) conserve energía (DiLaura, Houser, Mistrick Steffy, 2011). Sin embargo, durante las últimas décadas, la evidencia empírica ha demostrado que muchos aspectos de la fisiología humana y el comportamiento son influenciados por la iluminación que impacta en la retina (Wetterberg, 1993; Erren, Reiter \& Piekarski, 2003; Refinetti, 2016). En 2002 se produjo el descubrimiento de un nuevo fotoreceptor especializado en la retina, las células ganglionares retinales intrínsecamente fotosensitivas (ipRGCs) cuyo fotopigmento es la melanopsina (Berson, Dunn \& Takao, 2002). Estos receptores son el principal input del sistema circadiano e inician el camino no visual de la luz para la sincronización del reloj biológico, ubicado en el hipotálamo. Según Brainard \& Hanifin (2017 pp 830), la confirmación de que la luz regula la fisiología circadiana, neuroendocrina y neuro-comportamental humana y su influencia en la salud y el bienestar, es un avance científico que transformará la iluminación arquitectónica, con un impacto esperado comparable a las tecnologías de iluminación de estado sólido (LED). El aporte original al conocimiento de este proyecto radica en el desarrollo de indicadores de EEAE de los efectos no visuales de la luz en espacios interiores desde una perspectiva ergonómica, obteniéndose un doble beneficio desde la salud y la sustentabilidad.

Es en este marco que se desarrolla la tesis doctoral titulada Ergonomía Ambiental e Iluminación Integrativa. Desarrollo de créditos para un Sistema de Etiquetado Energético-Ambiental de Edificios No Residenciales que se describe en la próxima sección.

\section{Desarrollo}

El objetivo general de este proyecto (OG) es Incorporar a los Factores Humanos y la Ergonomía como créditos de EEAE. Por su parte, los objetivos específicos (OE) son OE1: Analizar los distintos sistemas de EEAE, en particular los créditos específicos de Iluminación, y sus beneficios asociados en términos de Ergonomía Ambiental; OE2: Seleccionar y/o Desarrollar indicadores específicos de iluminación saludable considerando: (i) exposición a luz biológicamente activa, (ii) acceso a iluminación natural y visión al exterior; OE3: Proponer y validar un método de acreditación de iluminación saludable en edificios no residenciales (i.e. operacionalización de los indicadores del OE2, selección de métodos e instrumentos de verificación, determinación de escalas cuantitativas/cualitativas de puntuación y sus respectivos puntos de corte). La Hipótesis General que sustenta a esta investigación es que El desarrollo de créditos específicos de Iluminación Saludable para el EEAE permitiría caracterizar, cuantificar, evaluar, y proyectar/remediar entornos construidos que propicien el ahorro energético sin detrimento de la calidad ambiental interior, favoreciendo un desempeño confortable, saludable, eficaz y eficiente por parte de los usuarios.

Para la consecución de estos objetivos, se planificaron las siguientes actividades y metodologías:

1- Búsqueda de antecedentes: (i) En relación con los métodos de EEAE en general y en particular los capítulos referidos a iluminación, diferenciando aquellos que otorguen puntuación a aspectos Ergonómicos relacionados con el confort y los efectos visuales y no visuales de la luz; (ii) En relación a las variables dependientes de esta investigación (i.e. confort visual, rendimiento visual y cognitivo, iluminación circadiana)

2- Análisis de créditos de iluminación de EEAE: En base a los resultados obtenidos en (1) se aplicarán los criterios de inclusión/exclusión de Fowler \& Rauch (2006). Se realizará una valoración de estos créditos siguiendo los criterios de Nguyen \& Altman (2011), de cuya metodología de análisis se obtiene un puntaje de 0 a 100.

3- Propuesta de criterios de etiquetado de iluminación integrativa: Se trabajará a partir de los criterios de etiquetado del Standard Well V2-2018 y de los resultados obtenidos en (2) para los siguientes aspectos: Estimulación visual y circadiana, deslumbramiento, iluminación natural, control de la iluminación natural y artificial, acceso a la luz y visión al exterior.

4-Estudios de campo: Los criterios de iluminación saludable propuestos en (3) serán implementados en locales no residenciales del Área Metropolitana de Mendoza. En particular, se espera acceder a las bibliotecas públicas construidas por el Gobierno de 
Mendoza a través del Ministerio de Infraestructura y Energía desde 2012, que resultó en la construcción de 18 edificios idénticos (uno por departamento), que fueron diseñados bajo el criterio de la sustentabilidad energética. Cabe destacar que uno de estos edificios (en el departamento de Lavalle) ya fue abordado durante su especialización en Desarrollo Sustentable del Hábitat Humano. Se propone una muestra de cinco edificios de biblioteca en el desarrollo de esta investigación. Se espera contrastar los resultados con locales destinados al mismo uso sin criterios de sustentabilidad energética. Este estudio de caso-control incluirá la caracterización de: (i) entorno físico y luminoso (Protocolo PC-SRT, Ev, Ec, Eh), Mapeos de luminancia por HDRI, y sus los efectos en los ocupantes de cada entorno luminoso; (ii) Análisis de deslumbramiento (GSV, DGP, UGR), (iii) caracterización energética (método LENI) y ergonómica (Método ELI), así como (iv) Percepción de la Calidad Ambiental de la Iluminación interior por medio de instrumentos subjetivos validados.

\section{5- Análisis de datos, conclusiones y redacción del Manuscrito.}

\section{Avances}

En esta primer etapa se está avanzado en el estudio del ecosistema de sistemas de Etiquetado-Energético Ambiental de Edificios (EEAE), para determinar una taxonomía de las mismas, conociendo sus características, puntos en común y diferencias. Los términos NORMA o ESTANDAR / CERTIFICACIÓN / GUÍAS o RECOMENDACIONES DE BUENAS PRÁCTICAS suelen utilizarse indistintamente, sin embargo el análisis realizado permite dar precisiones en cuanto a su significado, alcance y campos de aplicación.

ESTANDAR/NORMA: La Guía ISO/IEC 2:1996 define una norma como un documento, establecido por consenso y probado por un organismo reconocido, que proporciona, para un uso común y repetido, reglas, directrices o características para actividades o sus resultados, con el fin de conseguir un grado óptimo de orden en un contexto dado. Dentro de los organismos que imparten normas podemos nombrar ISO - IRAM - ASHRAE -ASTM. Disposiciones de carácter técnico, para ecología, eficiencia energética, de construcción, entre muchas. Las normas pueden ser de carácter, nacional (e.g. IRAM Instituto Argentino de Normalización y Certificación), regional (e.g. EN Norma Europea), o internacional (e.g. ISO Organización Internacional de Normalización)

Las normas tienen que ser elaboradas por un organismo reconocido de normalización. Además de los organismos nacionales de estandarizaión, existen asociaciones profesionales, como por ejemplo la IEEE (electrónica), o la CIE (iluminación), que elaboran estándares, y que son tomadas por organismos de normalización dando lugar a normas ISO- IEEE, o ISO- CIE. Es aquí donde surge la diferencia entre una norma y un estándar. Toda norma es un estándar, pero no todos los estándares son normas. En el primer caso, estamos frente a un estándar de iure mientras que en el segundo se considera estándar de facto.

CERTIFICACIÓN: Es la provisión por parte de un organismo independiente de garantía por escrito (un certificado) de que el producto, servicio o sistema en cuestión cumple con requisitos específicos, por ejemplo una norma o un estandar.

ACREDITACIÓN: Es el reconocimiento formal por parte de un organismo independiente, generalmente conocido como organismo de acreditación, de que un organismo de certificación opera de acuerdo con estándares internacionales.

ETIQUETADO: Es una forma de certificación, donde se trabaja con una ponderación de criterios cuyo grado de cumplimiento determina diferentes categorías ordinales. El etiquetado puede asociarse a estandares de uire como la norma IRAM 11900 de etiquetado de eficiencia energética de viviendas, por ejemplo. En ese marco, la Etiqueta de Eficiencia Energética es un documento en el que figura la Clase de Eficiencia Energética, (escala de letras, desde la "A" hasta la "G") asociada a un rango de valores del Índice de Prestaciones Energéticas (IPE). La etiqueta es una herramienta que permite conocer de manera rápida y fácil, el consumo de energía y cuál es su nivel de eficiencia energética. Permitirá determinar un "índice de prestación energética" (IPE) para clasificar la eficiencia del inmueble con su unidad de medida $\mathrm{kWh} / \mathrm{m} 2$ al año. El etiquetado también puede asociarse a estándares de facto, desarrolladas y gestionadas por organizaciones públicas y no gubernamentales, con o sin fines de lucro. Algunos ejemplos son:

- $\quad$ LEED (sigla de Leadership in Energy \& Environmental Design) es un sistema de certificación de edificios sostenibles, desarrollado por el Consejo de la Construcción Verde de Estados Unidos (US Green Building Council).

- WELL Building Standard es un sistema de puntuación para edificios que mide su impacto en la salud y el bienestar de las personas. 
- BREEAM (Building Research Establishment Environmental Assessment Methodology) es el primer método de evaluación y certificación de la sostenibilidad en la edificación.

- PASSIVHAUS o casa pasiva, es un estándar de construcción nacido en Alemania en 1991.

- CASBEE Comprehensive Assessment System for Built Environment Efficiency, Sistema de evaluación integral para la eficiencia del entorno construido.

\section{MÉTODO}

ACV Análisis del Ciclo de Vida es una evaluación de impacto ambiental de productos, procesos y actividades. Tiene en cuenta fundamentalmente impactos asociados al consumo y a las emisiones a lo largo del todo el ciclo de vida y la cadena de suministros de un proceso, de un producto o de una actividad. Es decir que considera desde la extracción de las materias primas, para el caso de un producto, el pre procesamiento, la distribución, el consumo y su fin de vida. Esta metodología está estandarizada bajo la serie 14000 de las Normas ISO que es de gestión ambiental. Específicamente en la 14040 y la 14044. Dando las mismas los principios básicos y los lineamientos para llevar adelante un estudio de análisis de ciclo de vida. Fundamentalmente el resultado que se obtiene es el de un perfil ambiental de ese producto, proceso o actividad. Recientemente se han incorporado los estudios de ciclo de vida de las organizaciones. Dentro de las metodologías de impacto ambiental, lo que nos provee el análisis de ciclo de vida es una visión holística porque tiene en cuenta no solamente más de un impacto a la vez y considera todas las etapas del ciclo de vida de un producto.

Permite realizar comparaciones objetivas considerando todas las fases que un sistema sigue durante su vida útil: construcción, uso y desmantelamiento final. Dentro del ACV existen distintos métodos de huellas, según la variable relevante que se tome como indicador (emisiones de $\mathrm{CO} 2$, consumo de agua).

\section{INDICADORES}

Los indicadores que son aquellos que miden el comportamiento de las variables y deben estar vinculados a cada componente probado. Y por otro lado están los índices, estos pueden ser el resultado de un grupo de indicadores. Los índices son más complejos que los indicadores, ya que se basan en ecuaciones o modelos matemáticos. Por el contrario, los índices nos permiten asignar un valor a cualquier aspecto medido.

Las huellas son indicadores de sustentabilidad de un solo aspecto, evaluando la contribución de ese proceso, producto o actividad, indicando el impacto asociado a las emisiones de gases de efecto invernadero o al consumo de agua.

HUELLA ECOLÓGICA (del inglés ecological footprint) es un indicador del impacto ambiental generado por la demanda humana que se hace de los recursos existentes en los ecosistemas del planeta, relacionándola con la capacidad ecológica de la Tierra de regenerar sus recursos.

HUELLA DE CARBONO indicador ambiental que pretende reflejar la totalidad de gases de efecto invernadero emitidos por efecto directo o indirecto de un individuo, organización, evento o producto.

HUELLA HIDRICA es un indicador medioambiental que define el volumen total de agua dulce utilizado para producir los viene y servicio que habitualmente consumimos. Es una variedad necesaria que nos dice el agua que nos cuesta fabricar un producto.

ELI este indicador plantea una combinación de componentes objetivos y subjetivos, siendo los cinco criterios propuestos para la evaluación de la calidad de la iluminación.

\section{Conclusiones}

En lo avanzado hasta el momento vemos la existencia de numerosas metodologías de evaluación ambiental y de diversos actores relacionados a las mismas. Se realizó un glosario básico y una taxonomía preliminar como primer instancia para el desarrollo de créditos de iluminación integrativa para sistemas de EEAE que logre un balance entre requerimientos energéticos y ergonómicos. Con el avance del presente trabajo se analizarán los distintos sistemas consiguiendo un marco teórico para avanzar en la propuesta de un sistema de acreditación, la aplicación del mismo y su posterior validación. 


\section{Referencias}

Berson DM, Dunn FA, Motoharu Takao (2002). Phototransduction by retinal ganglion cells that set the circadian clock. Science; 295:1070-73.

Brainard C \& Hanifin JP (2017) Photoreception for Human Circadian and Neurobehavioral Regulation. En Karlicek R Handbook of Advanced Lighting Technology. Springer-Verlag

Brown C (2007). Eco-ergonomics. Proceedings of the New Zealand Ergonomics Society Conference 2007. Waiheke Island.

Cedeño-Laurent JG, Williams A, MacNaughton P, Cao X, Eitland E, Spengler J, \& Allen J (2018). Building Evidence for Health: Green Buildings, Current Science, and Future Challenges. Annual review of public health, 39, 291-308.

Cole J, Howard N, Ikaga T \& Nibel S (2005). Building Environmental Assessment Tools: current and future roles. In World Sustainable Building Conference (pp. 27-9).

DiLaura D, Houser K, Mistrick R \& Steffy G (2011). The lighting Handbook 10th edition: Reference and application. Illuminating Engineering Society of North America, 120. ISBN\#978-0-87995-241-9

Erren TC, Reiter RJ, Piekarski C (2003) Light, timing of biological rhythms, and chronodistuption in man. Naturwissenschaften, 90(11): 485-494.

Fowler KM, Rauch EM (2006). Sustainable Building Rating Systems Summary. Pacific Northwest National Laboratory - U.S. Department of Energy

Garcia-Acosta G, Saravia MH, Riba C (2012). Ergoecology: evolution and challenges. Work, 41(1): 2133-2140.

Gibberd J (2005). Assessing sustainable buildings in developing countries - the sustainable building assessment tool (SBAT) and the sustainable building lifecycle (SBL). In: Proceedings of the world sustainable building conference. Tokyo (pp. 1605-12).

Hanlon M (2007). World population becomes more urban than rural, accedido el 6/07/2018 en http://www.gizmag.com/go/7334/

Hedge A \& Dorsey JA (2013). Green buildings need good ergonomics. Ergonomics, 56(3), 492-506.

Hirning MB, Isoardi GL \& Cowling I (2014). Discomfort glare in open plan green buildings. Energy and Buildings, 70, 427-440.

Klepeis NE, Nelson WC, Ott WR, Robinson JP, Tsang AM, Behar JV, Hern SC, Engelman WH (2001). The National Human Activity Pattern Survey (NHAPS): A resource for assessing exposure to environmental pollutants. Journal of Exposure Analysis and Environmental Epidemiology, 11: 231-252.

Khoshbakht M, Gou Z, Lu Y, Xie X \& Zhang J (2018). Are green buildings more satisfactory? A review of global evidence. Habitat International.

Libovich A (2005). Assessing green building for sustainable cities. In: Proceedings of the world sustainable building conference. Tokyo (p.p. 1968-71).

Nguyen BK \& Altan H (2011). Comparative review of five sustainable rating systems. Procedia Engineering, 21, 376-386.

Moray N (1995). Ergonomics and the global problems of the twenty-first century. Ergonomics, 38(8): 1691-1707.

Refinetti R (2016) Circadian physiology. CRC press

Rodriguez R, Lasagno C, Monteoliva JM, Yamín J, Pattini A (2017) Ergonomía Verde: Factores humanos en la iluminación natural sustentable. XIII Jornadas Argentinas de Luminotecnia. San Rafael, Argentina.

Saravia-Pinilla MH, Daza-Beltrán C, García-Acosta G (2016). A comprehensive approach to environmental and human factors into product/service design and development. A review from an ergoecological perspective. Applied ergonomics, 57: 62-71.

Sev, A. (2011). A comparative analysis of building environmental assessment tools and suggestions for regional adaptations. Civil Engineering and Environmental Systems, 28(3), 231-245. https://doi.org/10.1080/10286608.2011.588327

Schweizer C, Edwards RD, Bayer-Oglesby L, Gauderman WJ, Ilacqua V, Jantunen MJ (2007). Indoor time microenvironment-activity patterns in seven regions of Europe. J. Exposure Science \& Environmental Epidemiology, 17(2): 170-181.

Stern N (2006). Stern Review on the Economics of Climate Change. London: HM Treasury

Thatcher A (2013). Green ergonomics: definition and scope. Ergonomics, 56(3): 389-398. Thatcher A \& Milner K (2014). Changes in productivity, psychological wellbeing and physical wellbeing from working in a 'green' building. Work, 49(3), 381-393.

Vagge CS \& Czajkowski JD (2012). Impact of the application of Energy Efficiency 13059 Act in relation to the new Land Use Ordinance of the city of La Plata and the Building Labelling Standard IRAM 11900. Ambiente Construído, 12(2), 23-35.

Wetterberg L (1993) Light and Biological Rhythms in Man. Pergamon Press

Zink KJ \& Fischer K (2013). Do we need sustainability as a new approach in human factors and ergonomics? Ergonomics, 56(3): 348-356. 